\title{
Short Introduction
}

Set-up and delivery of a clinical research project can be complicated and difficult. We introduce the regulatory processes involved in gaining approval for clinical research and discuss the obstacles that may be encountered.

\section{Main Introduction}

The aim of this article is to introduce key processes involved to gain approval for clinical research and describe some important obstacles faced by researchers. It should be noted that regulatory requirements depend upon both the specifications of the project and local policies. England and devolved administrations within the UK have different operating practices.

A number of significant changes to research regulation are being implemented, as part of a drive to streamline and improve the set-up and delivery of clinical research studies.

\section{The Basics}

It is important not to underestimate the amount of preparatory work, time and support needed to undertake a clinical research project. Significant groundwork is needed even before a study is ready for research approval application.

The basic initial requirements include a research protocol, secured funding, sponsorship (box 1) and adequate indemnity arrangements. In addition, the specific approval requirements of the research project need to be established early on, as the approvals processes can be complicated and longwinded. Applications to most regulatory bodies are made through the Integrated Research Application System (IRAS), the national online research approvals and permissions application system

The typical applicant requires both ethical approval and NHS site approval ('NHS permission' or 'Research and Development (R\&D) approval') for their study. Ethical approval is obtained through the Research Ethics Service (RES) for all studies involving NHS patients or NHS property. The ethics review process is performed by research ethics committees (RECs). Where studies are led from England, both ethical and NHS site approval are arranged through a single system called 'Health Research Authority (HRA) approval'. For devolved administrations, alternative applications arrangements are in place.

Certain studies require Medicines and Healthcare Regulatory Agency (MHRA) approval. The MHRA is an agency within the Department of Health. Clinical trial authorisation (CTA) is issued by the MHRA for clinical trials of medicinal products (MHRA Medicines Regulation). Clinical investigations of non-CE marked medical devices also require MHRA review (MHRA Medical Devices Regulation). Other regulatory bodies issue approvals in specialised circumstances such as the Human Tissue Authority (HTA), Human Fertilisation and Embryology Authority (HFEA) and the Gene Therapy Advisory Committee (GTAC), which acts as the REC for gene therapy clinical research. 
Supporting documents are submitted with applications and include participant information sheets and consent forms. Guidance for preparing these forms can be found at www.hra.nhs.uk

The project will be scrutinised by a number of bodies to ensure that the study design is ethically and scientifically sound, there has been adequate resource planning, the burdens and costs are appropriate and accurately represented and research governance standards are followed. The current (2005) Research Governance Framework (box 2) provides goodpractice guidance to researchers. This will be replaced in due course by a new UK-wide framework for health and social care research.

\section{The Health Research Authority}

The Health Research Authority (HRA) is an NHS body, launched in December 2011 to improve and streamline health research regulation whilst promoting and protecting the interests of patients and the public (www.hra.nhs.uk). It was set up following commitments made in the Government's 'Plan for Growth' (2011) to tackle failings in the initiation and delivery of health research in the UK. This came in response to a Government -commissioned report by the Academy of Medical Sciences 'A new pathway for the regulation and governance of health research' (2011) which proposed, amongst other measures, creation of a new health research regulatory agency.

The HRA oversees the Research Ethics Service (RES), which manages ethical review for clinical research in the UK. The functions of RES are to "protect the rights, safety, dignity and well-being of research participants" and "facilitate and promote ethical research that is of potential benefit to participants, science and society". Since its inception the HRA has also taken over provision of IRAS and has combined ethics committee with R\&D approvals into a single HRA assessment called 'HRA Approval'. This applies to studies led from England only, but the HRA works closely with devolved administrations to harmonise operating practices within the UK.

The HRA has produced a draft UK-wide Policy Framework for Health and Social Care Research to replace the current Research Governance Framework. At the time of writing, this has been through a public consultation period and is expected to come into force, after revisions, sometime in 2016.

In line with its objectives, the HRA has close links with other bodies involved in research regulation and promotion such as the MHRA and National Institute for Health Research (NIHR).

\section{IRAS}

The Integrated Research Application System (IRAS) is a freely accessible online system that is used to prepare and submit applications for healthcare research approval. The website 
address is www.myresearchproject.org.uk. An IRAS account is simple to set up with an email address and chosen password. Multiple projects can be entered within a single account.

Resources available to guide researchers within IRAS include an e-learning module and help page. Green icons attached to questions within the forms provide question-specific guidance. A telephone contact number and email address is also available for technical support and other queries.

When a new project is created, it opens to a navigation page. On the left side of the page is an access panel for all the forms that can be captured in IRAS. For each project, information entered into the 'Integrated Dataset' of IRAS allows easy population of other forms within the IRAS system and avoids the need to enter duplicate information for different approvals.

IRAS is designed to tailor further questions according to the responses given. Care is therefore needed when answering questions in the project filters. An incorrect answer can mean the subsequently generated questions are not appropriate for the study.

Responses to the project data questions in IRAS should be carefully considered prior to project submission for regulatory approval. A selection of typical project data question topics is shown in box 3(UK Health Departments (2011), World Health Organisation 2010)).

\section{Ethical review}

Applications for ethical approval are submitted in IRAS and are reviewed by RECs. Each REC consists of lay members and people with particular expertise including healthcare professionals and academics.

Most REC applications in the UK are processed through a new Central Booking Service (CBS), which replaces the Central Allocation System, Local Allocation System and Proportionate Review Allocation System*. It is still possible to apply to a specific REC of choice using the new service.

The application for REC review is booked by telephone. Contact details are available on the HRA website www.hra.nhs.uk. Electronic submission of all necessary documents through IRAS is required on the same day as booking. Before booking it is therefore important to have all supporting documentation ready and ensure that appropriate authorisations have been made. Mandatory electronic authorisations have been introduced, meaning that all required signatures must be performed electronically through the IRAS system. All signatories will therefore need to have their own IRAS account in order to authorise. All required authorisations must be made before the application is submitted.

It is recommended that applicants attend the REC meeting as it allows committee members to address queries directly with the researcher.

With few exceptions, the REC must provide an opinion within 60 days of receipt of a valid application. Aside from a 'favourable' or 'unfavourable' opinion, a 'favourable with additional conditions' response may be issued. Alternatively the opinion can be provisional, 
pending further information or advice from a specialist referee. 'No opinion' may arise in a proportionate review case, with a recommendation for referral to a full meeting.

*Proportionate review was introduced to speed up processing of applications that are thought to pose low risks to participants. Guidelines for eligibility are available on the HRA website, using the No Material Ethical Issue Tool (NMEIT).

\section{NHS permission}

Research taking place within an NHS organisation must be approved by the host NHS organisation before it starts. Until recently, separate NHS permissions for each site were requested using Site-Specific Information (SSI) forms generated through IRAS. Applications in England are still made through IRAS but coordinated through the HRA. Separate coordinated system arrangements have been developed for each devolved administration in the UK.

In England, each site is now asked to make a local 'capacity and capability' assessment. If its involvement in the study is then jointly agreed, arrangements are put in place to fulfil its requirements. The final step is confirmation that it can proceed. For non-commercial studies, the HRA is trialling 'Schedule of Events' and 'Statement of Activities' template documents for submission through IRAS, which are intended to clarify the resource requirements and cost implications for each site. Costs for activities are recorded in line with the Department of Health AcoRD guidelines (box 4).

Prior to the introduction of the HRA Approval system it was possible for certain studies in England to request NHS permission through the NIHR Coordinated System for gaining NHS Permission (CSP). This service was provided by the NIHR Clinical Research Network (CRN), as part of its remit to support high quality research studies (www.crn.nihr.ac.uk). This role has been taken over by the HRA, but those studies adopted by the CRN continue to be eligible for extra practical support through NIHR (box 5).

\section{Obstacles to approval}

The UK has become a less attractive location for conducting clinical research, with evidence of a significant fall in the number of clinical trials undertaken. This has been largely attributed to difficulties in navigating processes for regulatory approvals (4). Excessively complex legislation, over-stringent and inconsistent interpretation of regulations and inadequate support of research are amongst the key obstacles identified. In response to these concerns, changes in regulatory mechanisms are being implemented.

\section{Obtaining NHS permission}

It is widely agreed that obtaining NHS permission has been one of the greatest obstacles to conducting a clinical trial in the UK (House of Commons Science and Technology Committee (2013-2014)), and widely reported as an important source of delay for multicentre study set-up (Al-Shahi R (2005), Mallick Andrew A et al (2009), Rees M et al (2010), Thompson AGH et al (2010), Snooks H et al (2012)). This is partly attributed to a cumbersome system of duplicate applications to every participating site, each with its own 
internal arrangements for approving research. The requirements are not always transparent and applications can take a significant length of time to process.

England, Scotland, Wales and Northern Ireland have developed separate coordinated systems designed to improve the process of gaining NHS permission, especially across multiple sites. In England, the HRA has taken on the responsibility of providing a single sign-off for NHS centres participating in health research studies.

The NIHR now publishes outcome data for clinical trials, which include a 70 day timeline from receipt of a valid research application to recruitment of the first patient.

\section{Research culture within the NHS}

Clinical research in the UK would benefit from a culture change; ensuring research is treated as a "core activity" within the NHS (Cancer Research UK (2010)). Resistance and apathy towards research in the NHS can jeopardise the entire research process from project approvals to study completion. Obstacles can arise in many areas including concerns about research costs or cost recovery and diversion of resources and staff time. Other factors include a lack of understanding of research processes and benefits; mistrust of research, with a paternalistic approach discouraging patient involvement; and simply lack of incentive to support research projects. Some researchers are also struggling with lack of expertise and inadequate training in navigating the approval process, further compounding problems achieving project sign-off.

The Secretary of State, clinical commissioning groups and NHS England are required under the Health and Social Care Act 2012 (National Archives) to promote research and the use of research evidence. NHS England's draft document: Research and Development Strategy 2013-2018 "Research is everybody's business" (NHS England (2013)), sets out aims and objectives to help achieve this. It isn't entirely clear how the ambition to embed research into the health system will be realised and there is no quick fix. Addressing the bureaucratic obstacles, providing education and training, creating incentives to engage with research and encouraging involvement of patients and the public with research are important steps in the right direction.

\section{The European Clinical Trials Directive (CTD)}

Introduction of the Directive 2001/20/EC (European Commission (2001)) for clinical trials of investigational medicinal products has compromised clinical research activity in the UK by increasing costs and administration and extending the time required for launching new trials (Science and Technology Committee). This has been exacerbated by gold plating of EU legislation in the UK. The directive, drafted by the European Commission and enforced in the UK from 2004, aimed to improve regulation across the European Union and is acknowledged to have had favourable impact on the ethical regulation of trials in Europe. The CTD has since been revised in response to calls for more proportionate regulation, with largely positive reception.

Regulation (EU) No 536/2014 on clinical trials on medicinal products for human use (European Parliament (2014)) now replaces Directive 2001/20/EC. It includes the provision of a new EU portal and clinical trials database. The regulation will not be applied until the 
portal and database are independently assessed as being fully functional. At the time of writing this is predicted to be by the end of October 2018 .

\section{Conclusion}

Healthcare research regulation and governance in the UK is overly bureaucratic, complicated and confusing. These difficulties are recognised and there are ongoing efforts to streamline approval applications and improve support so that good clinical research can continue. Some progress has already been made, in particular for ethics approval application, which was once considered a significant bureaucratic stumbling block. Measures introduced in recent years include the introduction of 'multicentre' RECs, removing the need for multiple duplicate applications; clear timelines for provision of a REC opinion; and introduction of IRAS. It is hoped that new measures such as the HRA Approval system will similarly improve the process of gaining NHS permission. It is clear that further work is needed. The various approvals processes should run in parallel and be overseen by suitably qualified and trained authorities who have an understanding of the regulatory/ legal/ technical aspects of the project. This would ensure greater consistency in approach, reduce unnecessary bureaucracy and help guide rather than hinder the researcher.

Checks and controls must exist in research but it is important to ensure that regulatory scrutiny is proportionate to risk. The challenge is to ensure that the protection of the rights of trial participants is prioritised whilst avoiding excessive bureaucracy. However, the drive to improve efficiency should enhance rather than compromise ethical standards. Patients and the public are let down by missed opportunities to participate in good research. It is also unethical to squander research funding, waste research time and ultimately fail to answer important clinical questions because of red tape and unnecessary constraints.

In addition to addressing excessive bureaucracy at national and local levels, a new culture in the NHS must be fostered that treats research as a core responsibility rather than simply a danger or a nuisance.

\section{References}

1. Academy of Medical Sciences (2011). A new pathway for the regulation and governance of health research.http://www.acmedsci.ac.uk/policy/policy-projects/a-new-pathway-for-theregulation-and-governance-of-health-research/

2. Al-Shahi R (2005). Research ethics committees in the UK- the pressure is now on research and development departments. J R Soc Med 98: 444-447

3. Cancer Research UK submission to the Academy of Medical Sciences (AMS) review of the regulation and governance of medical research June 2010.

http://www.cancerresearchuk.org/prod_consump/groups/cr_common/@nre/@pol/documents/ generalcontent/cr_053410.pdf

4. Department for Business Innovation \& Skills (2011).The Plan for Growth. http://cdn.hmtreasury.gov.uk/2011budget_growth.pdf 
5. Department of Health (2005) Research Governance Framework for health and social care: Second edition.

https://www.gov.uk/government/publications/research-governance-framework-for-healthand-social-care-second-edition

6. European Commission. EU Clinical Trials Directive

2001/20/EC.http://ec.europa.eu/health/human-use/clinical-trials/index_en.htm

7. European Parliament, Council of the European Union (2014). Regulation (EU) 536/2014 of the European Parliament and of the Council of 16 April 2014 on clinical trials on medicinal products for human use, and repealing Directive 2001/20/EC. Official Journal of the European Union 27 May 2014; L158: 1-76

8. House of Commons Science and Technology Committee Clinical trials Third Report of Session 2013-14

http://www.publications.parliament.uk/pa/cm201314/cmselect/cmsctech/104/104.pdf

9. Mallick Andrew A, O’Callaghan Finbar JK (2009)Research governance delays for a multicentre non-interventional Study R Soc Med 102: 195-198

10. MHRA medicines regulation.

http://www.mhra.gov.uk/howweregulate/medicines/index.htm

11. MHRA medical devices regulation.

http://www.mhra.gov.uk/howweregulate/devices/index.htm

12. National Archives. Health and Social Care Act 2012.

http://www.legislation.gov.uk/ukpga/2012/7/contents

13. NHS England Research and Development Strategy 2013 - 2018 draft document Dec 2013. http://www.england.nhs.uk/wp-content/uploads/2013/12/nhs-england-res-stratconsult.pdf

14. Rees M, Wells F. Falling Research in the NHS. A clear national strategy is needed to overcome local barriers to research.

BMJ 5 June 2010 Vol 340. Editorials

15. Science and Technology Committee. Written evidence submitted by NHS European Office.http://www.publications.parliament.uk/pa/cm201314/cmselect/cmsctech/104/104vw28 .htm

16. Snooks H, Hutchings H, Seagrive A, Stewart-Broen S, Williams J, Russell I (2012).

Bureaucracy stifles medical research in Britain: a tale of three trials. BMC Medical Research Methodology 12: 122

17. Thompson AGH, France EF (2010). One stop or full stop? The continuing challenges for researchers despite the new streamlined NHS research governance process. BMC Health Services Research 10: 124 
18. UK Health Departments 9 May 2011 (Updated April 2012). Governance arrangements for research ethics committees: a harmonised edition.

https://www.gov.uk/government/publications/health-research-ethics-committees-governancearrangements

19. World Health Organisation (2010). Standards and Operational Guidance for Ethics Review of health-Related Research with Human Participants.

http://whqlibdoc.who.int/publications/2011/9789241502948_eng.pdf 